\title{
Prevalencia del Síndrome de Burnout en Estudiantes de Medicina
}

\section{Prevalence of Burnout Syndrome in Medical Students}

\author{
Jessica María González Escobar ${ }^{1}$ \\ Raphael Oliveira Ramos Franco Netto ${ }^{1}$ \\ Juliana de Almeida Rodrigues Franco Netto ${ }^{1}$ \\ Bruno Henrique Flores ${ }^{1}$ \\ Suelen Borges Andreo ${ }^{1}$ \\ Balbina Coronel de Bobadilla ${ }^{1}$ \\ ${ }^{1}$ Universidad Internacional Tres Fronteras-UNINTER, Facultad de Medicina. Pedro Juan Caballero- \\ Paraguay
}

\section{Resumen}

Introducción: El síndrome de Burnout en estudiantes de Medicina es la sensación de agotamiento, decepción y pérdida de interés por la actividad estudiantil.

Objetivo: Analizar la prevalencia del síndrome de Burnout entre los estudiantes de Medicina.

Materiales y Métodos: Estudio cuantitativo, observacional, de corte transversal, en el cual se obtuvo informaciones a partir de un cuestionario aplicado a los alumnos del $1^{\circ}$ al $4^{\circ}$ año de la Carrera de Medicina de la Universidad Internacional Tres Fronteras - UNINTER - Paraguay.

Resultados: El 6\% de los estudiantes no tiene síntomas del síndrome, 40\% tiene la posibilidad de desarrollarlo, $42 \%$ presenta una etapa temprana del síndrome, $9 \%$ se encuentran con el síndrome instalado y $3 \%$ está en fase de agotamiento. Se observó un porcentaje mayor de la presencia de síntomas entre los alumnos del segundo y tercer año.

Conclusión: Se percibe que un importante porcentaje de estudiantes de Medicina desarrollan la etapa temprana del síndrome de Burnout, por lo tanto, existe la necesidad de comprender mejor las causas de éste para así poder ayudar en la prevención del síndrome de Burnout en estudiantes de Medicina.

Palabras claves: Burnout, estudiantes de medicina, agotamiento.

\section{Abstract}

Introduction: The Burnout syndrome in medical students describes a state of exhaustion, disappointment and loss of interest in student activity.

Objective: To analyze the incidence Burnout syndrome among medical students. 
Methodology: A cross-sectional study in which information was obtained from a questionnaire applied to students from the 1st to 4th year of the course of Medicine of the International University Three Frontiers - UNINTER-PY.

Results: $6 \%$ of the students do not have symptoms of the syndrome, $40 \%$ have the possibility of developing it, $42 \%$ present an early stage of the syndrome, $9 \%$ find the syndrome installed and $3 \%$ are in exhaustion phase. A higher percentage of the presence of symptoms was observed among the second and tree year students

Conclusion: It is perceived that a considerable percentage of medical students present early symptoms of Burnout syndrome, therefore there is need to better understand the causes of this and thus be able to help in the prevention of Burnout syndrome.

Keywords: Burnout, medical students, exhaustion..

Fecha de recepción: 06-03-2020

Fecha de aceptación: 30-03-2020

Correspondencia:

Dr. Raphael Oliveira Ramos Franco Netto raphael fn@Hotmail.com

Universidad Internacional Tres Fronteras-UNINTER. Pedro Juan Caballero- Paraguay

\section{Introducción}

El síndrome de Burnout, es un trastorno psíquico depresivo, precedido por un intenso agotamiento físico, es crónicos en el contexto de trabajo y puede afectar a todos los individuos de todas las áreas profesionales, teniendo particular incidencia en médicos y estudiantes de medicina (1-3).

Por mucho tiempo investigadores han ido ampliando su atención sobre el tema Burnout con el objetivo de describir lo que lo origina y favorece, como se manifiesta, que consecuencias acarrea para los profesionales y los que los rodean y, consecuentemente, cuales son las formas de prevenir, detectar precozmente y tratar(1-5).

El síndrome de Burnout todavía no posee una definición ampliamente aceptada, por lo tanto, la más utilizada es la de Maslach que lo presenta como un síndrome psicológico compuesto por agotamiento emocional, despersonalización y reducción de la realización personal que ocurre en individuos que experimentan relaciones interpersonales en el trabajo (2). La palabra "burnout" se traduce en estar quemado, o agotado (2).

Estudios han demostrado que Burnout puede acometer independientemente del sexo, edad o local de trabajo. Estas afirmaciones pueden ser corroboradas con hallazgos diagnósticos del síndrome en diversos profesionales como, policías, profesores, amas de casa, estudiantes y hasta desempleados $(6,7)$

Es importante destacar que los estudiantes de medicina están diariamente expuestos a factores estresantes como: dificultades de adaptación, excesiva carga de trabajo y los estudios y falta de tiempo para el ocio y la relación social, lo que favorece el acometimiento al síndrome de Burnout. Estos factores son efectivamente responsables por muchos cambios psicosociales que llevan al desenvolvimiento del estrés, depresión, entre otros, pudiendo llegar al suicidio.

Siendo así, el objetivo de este trabajo fue evaluar la prevalencia del síndrome de Burnout en estudiantes de medicina de la Universidad Internacional Tres Fronteras de la ciudad de Pedro Juan Caballero, Paraguay 


\section{Materiales y métodos}

Fue realizado un estudio cuantitativo, observacional, de corte transversal, cuya población estuvo compuesta por estudiantes de Medicina del $1^{\circ}$ al $4^{\circ}$ año, independientemente del sexo y la edad, de la Universidad Internacional Tres Fronteras - UNINTER - localizado en la ciudad de Pedro Juan Caballero, Paraguay.

Se escogió aleatoriamente 30 estudiantes en cada año, totalizando 120 participantes, en su mayoría, brasileños, quienes recibieron orientación verbal en sala de clase sobre la motivación del trabajo de investigación y cómo responder, en forma correcta, el cuestionario.

Como instrumento para investigación, se utilizó o Maslach Burnout Inventory - Student Survey (8). El cuestionario es compuesto de 16 preguntas. Los ítems son evaluados por la frecuencia con resultados de cero (nunca) a seis puntos (siempre/todos los días). Para tener el diagnóstico de síndrome de Burnout, el individuo precisó simultáneamente encontrarse por encima del percentil de 66 de los escores de agotamiento e incredulidad y abajo del percentil de 33 de los escores de realización.

Se solicitó la firma de la hoja de consentimiento libre y aclarado, respetando las normas éticas de la investigación. Fueron excluidos de la investigación a los alumnos que se negaron a participar, menores de edad. Las variables analizadas fueron, porcentaje de estudiantes que presentan alguna etapa del síndrome de Burnout, el año en el que más se desarrolla el síndrome.

Los datos fueran colectados en una planilla, tabulados en Excel y analizado usando la fórmula de frecuencia relativa y los resultados expresados en porcentaje.

\section{Resultados}

De los resultados obtenidos, 38\% (46) e los encuestados ya escucharon hablar sobre el Síndrome de Burnout y $62 \%$ (74) dijeron no conocer sobre el tema (Grafico 1).

\section{Gráfico 1. Relación de alumnos que dijeron conocer o no sobre el tema de síndrome de Burnout.}

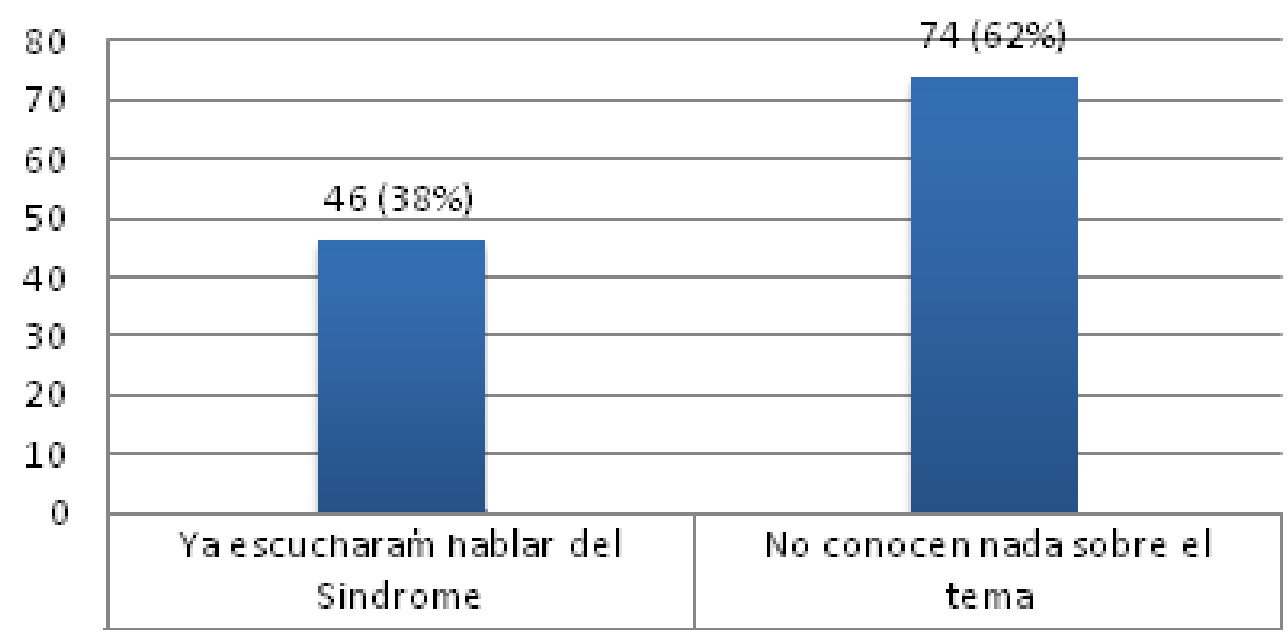

De los encuestados, según la puntuación y los síntomas de agotamiento psicológico 6\% (7) relataron no estar con ningún signo del síndrome o sea son considerados dentro del rango sin síndrome , 40\% (48) pueden ser considerados dentro del grupo de personas que están con la posibilidad de desarrollar el síndrome, 42\% (50) están con el síndrome de Burnout en una etapa temprana, 9\% (10) de 
los encuestados ya se puede considerar que están con el síndrome instalado y 3 (4) \% se encuentran en fase de agotamiento (Grafico 2).

Gráfico 2. Prevalencia general del síndrome de Burnout en los encuestados.

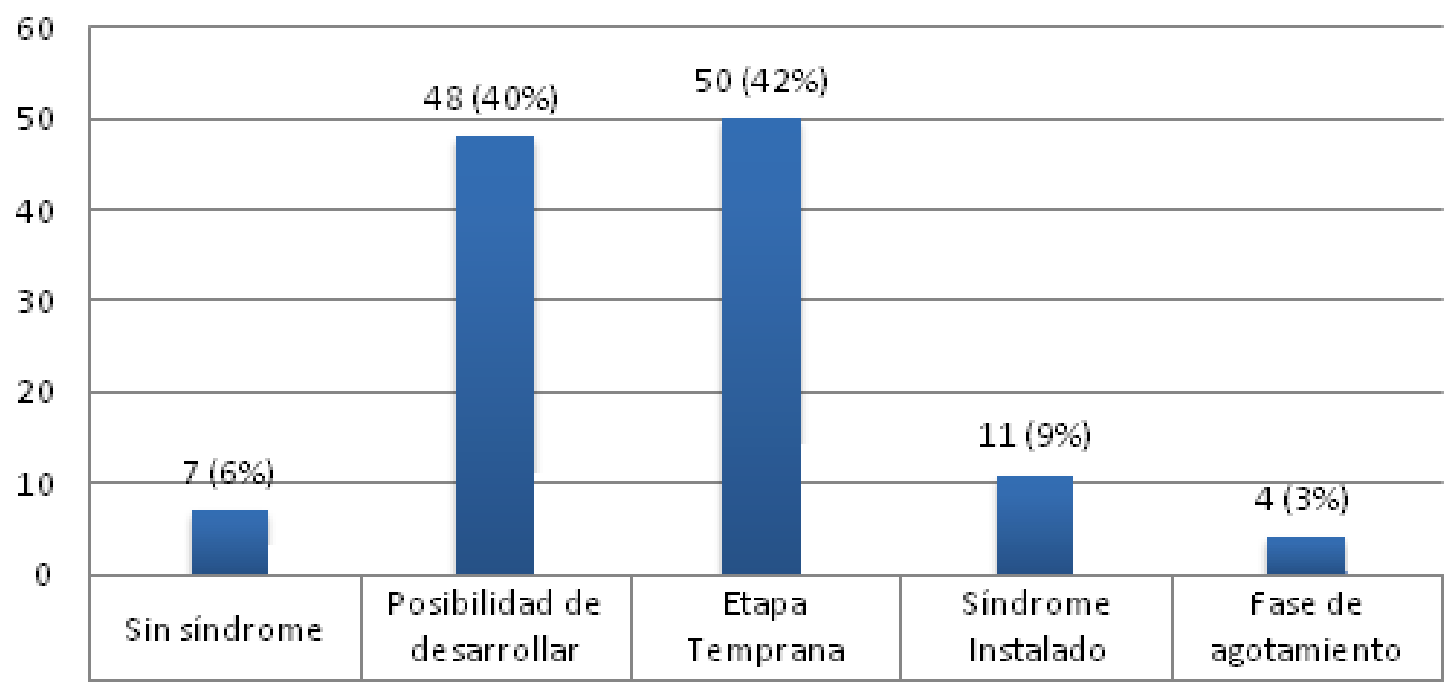

En la última encuesta también fue divida la puntuación y síntomas para verificar la prevalencia de alumnos de acuerdo con curso académico del $1^{\circ}$ al $4^{\circ}$ año, los resultados fueron los siguientes: $60 \%$ (18) de los alumnos del $1^{\circ}$ están en posibilidad de desarrollar, 53,3\% (16) de los alumnos del $2^{\circ}$ y $50 \%$ (15) de los alumnos del $3^{\circ}$ están en etapa temprana y $13,3 \%$ (4) de los alumnos del $4^{\circ}$ año están con síndrome instalado (Tabla 1).

Tabla 1. Distribución de los síntomas del síndrome de Burnout según el curso académico.

\begin{tabular}{|c|c|c|c|c|c|c|c|c|c|c|}
\hline \multirow{3}{*}{ Variable } & \multirow{2}{*}{\multicolumn{2}{|c|}{$1^{\circ}$ año }} & \multirow{2}{*}{\multicolumn{2}{|c|}{$2^{\circ}$ año }} & \multirow{2}{*}{\multicolumn{2}{|c|}{$3^{\circ}$ año }} & \multirow{2}{*}{\multicolumn{2}{|c|}{$4^{\circ}$ año }} & \multirow{2}{*}{\multicolumn{2}{|c|}{$\begin{array}{l}\text { Total } \\
\text { Estudiados }\end{array}$}} \\
\hline & & & & & & & & & & \\
\hline & $\mathbf{N}$ & $\%$ & $\mathbf{N}$ & $\%$ & $\mathbf{N}$ & $\%$ & $\mathbf{N}$ & $\%$ & $\mathbf{N}$ & $\%$ \\
\hline Sin síndrome & 3 & 10,0 & 2 & 6,7 & 1 & 3,3 & 1 & 3,3 & 7 & 6,0 \\
\hline $\begin{array}{l}\text { Posibilidad } \\
\text { desarrollar }\end{array}$ & 18 & 60,0 & 8 & 26,7 & 10 & 33,3 & 12 & 40,0 & 48 & 40,0 \\
\hline Etapa temprana & 7 & 23,3 & 16 & 53,3 & 15 & 50,0 & 12 & 40,0 & 50 & 42,0 \\
\hline Síndrome instalado & 2 & 6,7 & 3 & 10 & 2 & 6,7 & 4 & 13,3 & 11 & 9,0 \\
\hline Fase de agotamiento & 0 & 0 & 1 & 3,3 & 2 & 6,7 & 1 & 3,3 & 4 & 3,0 \\
\hline Total por año & 30 & 100,0 & 30 & 100,0 & 30 & 100,0 & 30 & 100,0 & 120 & 100,00 \\
\hline
\end{tabular}

\section{Discusión}

La Facultad de Medicina posee características únicas, que proporcionan un alto índice de estrés, influenciando la construcción de la identidad profesional del estudiante, junto al desenvolvimiento y acometimiento de Burnout, lo que apoya a su naturaleza ocupacional.

El modelo tradicional de enseñanza está asociado a: dificultad de adaptación en el inicio del curso, con cursos de nivelación y exámenes competitivos de acceso a la facultad; cambios de la 
enseñanza secundaria para una realidad de mayor responsabilidad y autonomía; frustración generada por el contacto tardío conla clínica, con el ciclo pre-clínico, insatisfacción con el curso y las estrategias de enseñanza; el deseo de desistir, motivado por la elevada sobrecarga de trabajo y sobre valorización de los métodos de evaluación muchas veces injustas $(3,4)$.

En el primer y segundo año, el Burnout surge fuertemente asociado al nivel de apoyo prestado por los profesionales de la facultad. Durante los primeros dos años del curso fue encontrado un porcentaje alto, con niveles moderados a elevados en el tercero y cuarto año. Estos números pueden estar relacionado en el exceso de trabajo, la falta de apoyo y la pérdida de autonomía y de control del ambiente de trabajo. Estos datos sugieren que puede ocurrir un agravamiento con la progresión para a clínica $(3,6,7,9)$.

Esta transición para el ciclo clínico puede ser considerada un momento importante, con elevada ansiedad, incertidumbres, expectativas y miedos. Todo esto, puede estar asociado al sentimiento de limitación a nivel del conocimiento científico, principalmente, con inicio del contacto directo con pacientes, muchos en estado grave, con mal pronóstico, aumentando la responsabilidad $(10,11)$.

Junto a esto, es importante destacar la elevada sobrecarga de trabajo y de contenidos teóricos presentes en la rutina de estudio, realización de rotaciones de horario muchas veces mal organizados, generando una preocupación y sensación de falta de control en la agenda, turnos nocturnos, alto índice de estrés, reducida confianza y experiencia, falta de tiempo para ocio, familia y amigos (6,9,12-14).

La insatisfacción, asociado a la sobrecarga de trabajo, evaluaciones, y la responsabilidad para con tratamiento de pacientes, podrán contribuir para elsentimiento de falta de preparación para ejercer la medicina y la insatisfacción relativamente al curso, con deseo de desistir $(3,14-16)$.

A pesar de la tendencia en el aumento de los niveles de Burnout en el ciclo clínico, algunos aspectos se mantienen durante todo el curso, tales como: mal ambiente general; sobrecarga de trabajo (muchas competencias a adquirir con recursos limitados de tiempo y memoria); no considerarse capaz de ser médico mismo cuando se tiene buen rendimiento; necesidad de posponer proyectos personales y la elevada presión social y académica relativamente al rendimiento escolar y al relacionamiento con compañeros y profesores (11,12,17-19).

Estudios, defienden que además de la enseñanza que se ha mantenido de forma semejante por décadas, es importante analizar la forma como la actual cultura médica favorece el Burnout, por facilitar el distanciamiento del médico en relación al paciente y a sí mismo. Así, el estudiante de medicina, dentro de este medio, está altamente vulnerable al Burnout. A medida que se distancia de sí mismo, podrá tener dificultades en cuidarse y desarrollar una identidad profesional madura y bien integrada, esto es, con valorización de si como un ser multidimensional y único, que ya existía y que va más allá del curso de medicina $(3,20)$

\section{Conclusión}

El estudio demostró una alta prevalencia de una etapa temprana del síndrome de Burnout entre los estudiantes, principalmente entre los alumnos del $2^{\circ}$ y $4^{\circ}$ año de la Carrera de Medicina. La presencia del síndrome sin acompañamiento psicológico puede traer consecuencias graves al estudiante, lo que demuestra la necesidad de una mejor comprensión de lo que es este síndrome y de los diferentes factores que inciden en la respuesta a la Carrera de Medicina, para proyectar y proceder a hacer actividades preventivas que ayuden al bienestar de los futuros médicos. De una forma general, La formación médica, parece promover médicos tecnicamente competentes, pero deficitários de algunas competencias humanas 


\section{Referencias bibliográficas}

1. Mansilla Izquierdo F. Maslach Burnout Inventory ( Mbi ). Maslach, C y Jackson, SE 1981; 1986. 1997;1-4.

2. Maroco J, Tecedeiro M. Inventário de burnout de Maslach para estudantes portugueses. Psicol Saúde Doenças [Internet]. 2009;10(2):227-35.

3. Costa EFDO, Santos SA, Santos ATRDA, Melo EV De, Andrade TM De. Burnout Syndrome and associated factors among medical students: a cross-sectional study. Clin (Sao Paulo) [Internet]. 2012;67(6):573-80.

4. Ishak W, Nikravesh R, Lederer S, Perry R, Ogunyemi D, Bernstein C. Burnout in medical students: A systematic review. Clin Teach. 2013;10(4):242-

5. Romani M, Ashkar K. Burnout among physicians. Vol. 9, The Libyan journal of medicine. 2014. p. 23556.

6. Santen SA, Holt DB, Kemp JD, Hemphill RR. Burnout in medical students: Examining the prevalence and associated factors. South Med J. 2010;103(8):758-63.

7. Swider BW, Zimmerman RD. Born to burnout: A meta-analytic path model of personality, job burnout, and work outcomes. J Vocat Behav. 2010;76(3):487-506.

8. Maslach C, Jackson SE, Leiter MP. Maslach Burnout Inventory. In: Evaluating Stress: A Book of Resources. 1997. p. 191-218.

9. Jennings ML. Medical student burnout: Interdisciplinary exploration and analysis. J Med Humanit. 2009;30(4):253-69.

10. de Abreu Santos ATR, Grosseman S, de Oliva Costa EF, de Andrade TM. Burnout syndrome among internship medical students. Med Educ.

11. Dahlin ME, Runeson B. Burnout and psychiatric morbidity among medical students entering clinical training: A three year prospectivequestionnaire and interview-based study. BMC Med Educ. 2007;7.

12. Silveira C, Norton A, Brandão I, Roma-Torres A. Saúde mental em estudantes do ensino superior: experiência da consulta de psiquiatria do centro Hospitalar São João. Acta Med Port. 2011;24:24756.

13. Rosales Ricardo Y, Rosales Paneque FR. [Towards a two-dimensional study of burnout syndrome in college students]. Cien Saude Colet. 2014;19(12):4767-75.

14. Backović D V., Živojinović Jl, Maksimović J, Maksimović M. Gender differences in academic stress and burnout among medical students in final years of education. Psychiatr Danub. 2012;24(2):175-81.

15. Enginyurt O, Cankaya S, Aksay K, Tunc T, Koc B, Bas O, et al. Relationship between organisational commitment and burnout syndrome: A canonical correlation approach. Aust Heal Rev. 2016;40(2):181-7.

16. Mori MO, Valente TCO, Nascimento LFC. Burnout Syndrom and Associated Factors Among Medical Students. Rev Bras Psiquiatr. 2012. In press

17. Schaufeli WB, Martinez IM, Pinto, AM., Salanova $M$, Bakker,AB. Burnout and engagement in university students. A Cross National Study. 2002; 33 (5): 464-481.

18. Gonçalves MB, Benevides-Pereira AMT. Consideraçõessobre o ensinomédico no Brasil: conseqüênciasafetivoemocionaisnosestudantes. Rev Bras.Educ Med. 2009; 33(3):493-504

19. Zuardi AW, Prota FDG, Del-Bem CM. Reduction of the anxiety of medical students after currcular reform. Rev Bras Psiquiatr. 2008; 30(2):136-8

20. Santos ATRA, Grosseman S, Oliva Costa EF, Andrade TM.Burnout syndrome among internship medical students. Med Educ. 2011 